\title{
Giant Left Atrium with a Large Thrombus
}

"Muhammad A. Sadiq ${ }^{1}$ and Hafidh A. Ba Omar²

$$
\text { أُّين أيسر عملاق به خثرة كبيرة }
$$

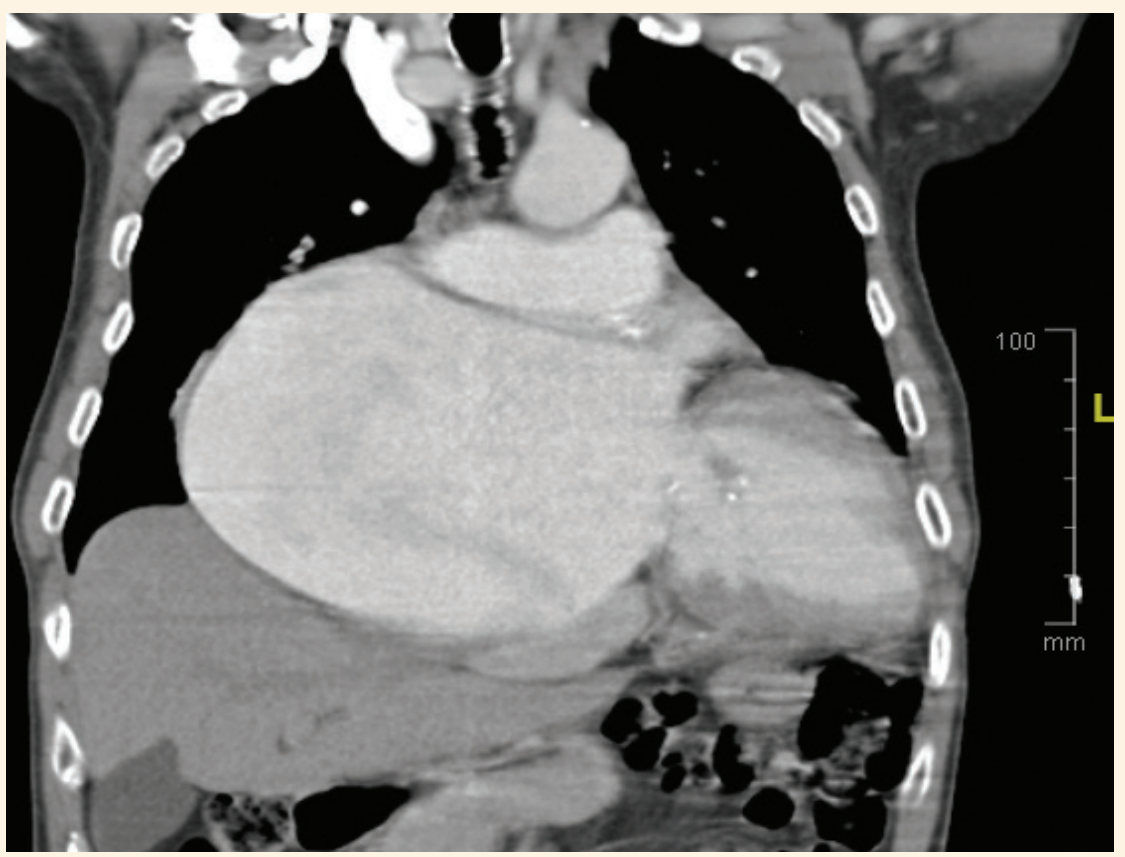

Figure 1: A non-contrast computed tomography two chamber view of the chest of a 73-year-old male patient showing a gigantic left atrium.

A 73-YEAR-OLD MALE PATIENT WITH KNOWN diabetes, hypertension and atrial fibrillation (AF) presented to a tertiary care centre with a transient ischaemic attack. He was referred to cardiology service for evaluation of his AF and to rule out a cardioembolic event. At the time of admission, the patient was on treatment with warfarin with an international normalised (INR) ratio of 2.5.

Transthoracic echocardiography revealed severe mixed mitral valve disease and giant left atrium (GLA) with profuse and dense whirling spontaneous echo contrast in the left atrium. There was a suspicion of a thrombus in the left atrium. Cardiovascular magnetic resonance $(\mathrm{CMR})$ imaging revealed a markedly dilated left atrium measuring $17 \times 11 \mathrm{~cm}$. There was a large filling defect at the left atrium's posterior aspect, which was not visualised adequately in CMR black blood sequence due to severe stagnation of blood. A non- contrast computed tomgraphy scan was performed, which showed a GLA with a large hypodense filling defect (thrombus) measuring $4.1 \times 3.6 \times 8.2 \mathrm{~cm}$ in its widest dimensions [Figures 1 and 2]. The patient's left ventricular ejection fraction measured by cardiac MRI was $49 \%$. He was offered mitral valve replacement (MVR) and left atrial volume reduction surgery, but he refused surgery.

\section{Comments}

GLA is a rare condition with a reported incidence of $0.3 \%{ }^{1}$ It is defined as an excessive dilatation of the left atrium by $>8 \mathrm{~cm} .{ }^{2}$ GLA is usually associated with rheumatic mitral pathology, but there are few reports of GLA in patients with non-rheumatic aetiology. ${ }^{1}$ It has been hypothesised that fibrosis with chronic inflammation combined with progressive 

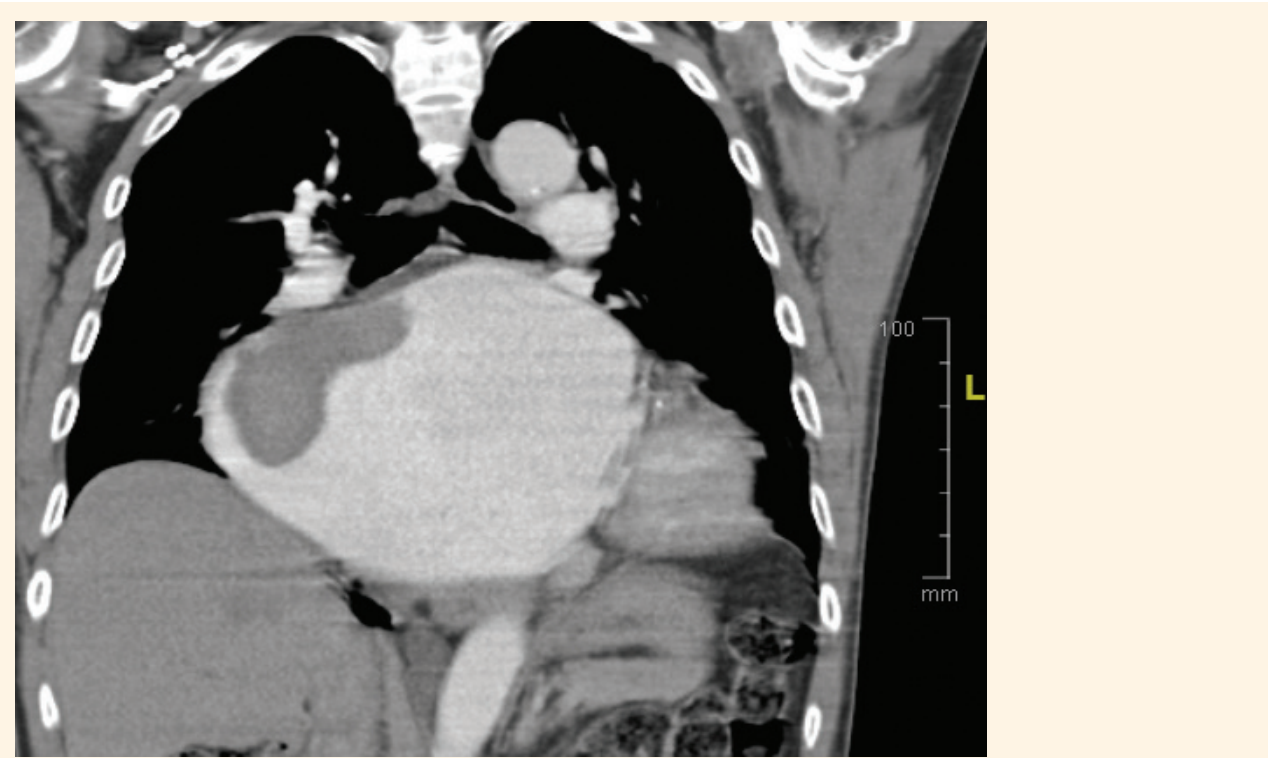

Figure 2: A non-contrast computed tomography two chamber view of the chest of a 73-year-old male patient showing the presence of a large thrombus in the left atrium.

atrial enlargement resulting from chronic volume and pressure overload is likely the cause of gross atrial enlargement.

Patients with GLA usually present with dyspnoea, dysphasia and thromboembolic events. The current patient complained of dyspnoea on minimal exertion, but there was no dysphagia. Although the risk of thromboembolism increases with LA dimensions and is independent of anticoagulants' administration, the published evidence of large thrombus formation in patients with LA size of $>8 \mathrm{~cm}$ is very scarce. There was a large LA thrombus formation in the current case despite having therapeutic INR with standard vitamin $\mathrm{K}$ antagonist therapy. There is a therapeutic dilemma when managing such patients. It is unknown whether the increase in the INR therapeutic range of $\geq 3.0$ will resolve GLA patients' clot. Contrary to current American Heart Association (AHA) guidelines that do not recommend using novel oral anticoagulants (NOAC) in patients with intracardiac thrombus and valvular AF, several reports are published on the use of off-label NOACs resulting in resolution of thrombus in patients with enlarged LA.,4 In a case report by Masarova et al., recurrent thrombus formation with therapeutic INR of 2.89 was observed in their patient with GLA after surgical removal of the thrombus. A combination of rivaroxaban and clopidogrel resulted in partial thrombus regression in their patient. ${ }^{4}$ Left atrial volume reduction surgery in AF patients with enlarged LA have shown superior freedom rates from AF up to five years compared with patients in the non-reduction group. ${ }^{5}$ AF plays a vital role in LA thrombus formation along with an increase in left ventricular (LV) volume overload and enlargement. Freedom from AF with LV volume reduction surgery may play an essential role in managing patients with GLA and large thrombus formation. The current patient was offered MVR, LA volume reduction surgery and thrombus removal, but he refused and was lost to follow-up.

\section{References}

1. El Maghraby A, Hajar R. Giant left atrium: A review. Heart Views 2012; 13:46-52.

2. Loch A, Sadiq MA, Ahmad WAW. Giant left atrium in a patient with prosthetic mitral valve. European Heart Journal 2013; 34:981.

3. Kirchhof P, Benussi S, Kotecha D, Ahlsson A, Atar D, Casadei B, et al. 2016 ESC Guidelines for the management of atrial fibrillation developed in collaboration with EACTS. Eur Heart J 2016; 37:2893-962.

4. Masarova L, Novak J, Pesl M, OndrasekJ, Semenka J, Simorova E, et al. Recurrent thrombus in the gigantic left atrium during effective anticoagulant therapy: Case report. BMC Cardiovasc Disord 2020; 20:86

5. Kim JH, Jang WS, Kim JB, Lee SJ. Impact of volume reduction in giant left atrium during surgical ablation of atrial fibrillation. J Thorac Dis 2019; 11:84-92. 